Wilfrid Laurier University

Scholars Commons @ Laurier

Psychology Faculty Publications

Psychology

$10-2-2007$

\title{
Urgency is a Non-monotonic Function of Pulse Rate
}

Frank A. Russo

Ryerson University, russo@ryerson.ca

Jeffery A. Jones

Wilfrid Laurier University, jjones@wlu.ca

Follow this and additional works at: https://scholars.wlu.ca/psyc_faculty

Part of the Psychiatry and Psychology Commons

\section{Recommended Citation}

Russo, F.A. \& Jones, J.A. (2007). The Journal of the Acoustical Society of America 122, EL185. doi: 10.1121/1.2784112.

This Article is brought to you for free and open access by the Psychology at Scholars Commons @ Laurier. It has been accepted for inclusion in Psychology Faculty Publications by an authorized administrator of Scholars Commons@ @aurier. For more information, please contact scholarscommons@wlu.ca. 


\title{
Urgency is a non-monotonic function of pulse rate
}

\author{
Frank A. Russo \\ Department of Psychology, Ryerson University, Toronto, Canada \\ russo@ryerson.ca \\ Jeffery A. Jones \\ Centre for Cognitive Neuroscience and Department of Psychology, Wilfrid Laurier University, Waterloo, Canada \\ jjones@wlu.ca
}

\begin{abstract}
Magnitude estimation was used to assess the experience of urgency in pulse-train stimuli (pulsed white noise) ranging from 3.13 to $200 \mathrm{~Hz}$. At low pulse rates, pulses were easily resolved. At high pulse rates, pulses fused together leading to a tonal sensation with a clear pitch level. Urgency ratings followed a nonmonotonic (polynomial) function with local maxima at 17.68 and $200 \mathrm{~Hz}$. The same stimuli were also used in response time and pitch scaling experiments. Response times were negatively correlated with urgency ratings. Pitch scaling results indicated that urgency of pulse trains is mediated by the perceptual constructs of speed and pitch.

(C) 2007 Acoustical Society of America PACS numbers: $43.66 . \mathrm{Ba}, 43.66 . \mathrm{Hg}$ [QJF]

Date Received: June 18, 2007 Date Accepted: August 5, 2007
\end{abstract}

\section{Introduction}

"The ranges of perception are ranges of time, and the time is subdivided by us, by the construction of our bodies and by our organs of perception. And since these modern means have become available, to change the time of perception continuously, from one range to another, from a rhythm into a pitch, or a tone or noise into a formal structure, the composer can now work within a unified time domain" (p. 95, Stockhausen, 1990/1971).

The effectiveness of an auditory warning is determined in part by its ability to convey urgency. Ideally, the warning will convey enough urgency so as to alert an observer and provoke some sort of action (Patterson, 1990). An auditory warning can be made more urgent in several ways, including increasing the pitch (frequency) and, in the case of warnings with multiple sound bursts, increasing the speed (i.e., decreasing the inter-onset time; Edworthy et al., 1991; Guillaume et al., 2003; Hellier et al., 1993). Research has conventionally framed these two influences on the experience of urgency as independent and their effects have been compared directly using psychophysical methods. For example, applying Stevens' power law, Hellier and Edworthy (1999) found that larger changes to pitch than to speed were necessary in order to yield equivalent changes in experienced urgency (slopes of 0.37 and 1.35 , respectively).

However, from a strictly temporal perspective, frequency and inter-onset time of auditory warnings may be interpreted as reciprocals of one another falling along different ends of the same rate-based continuum; that is, faster pulse rates (in terms of cycles per second or event onsets) tend to convey higher levels of urgency. Ecological psychoacoustics may provide a unifying framework for understanding rate-based predictors of urgency (Neuhoff, 2004). Specifically, it may be argued that it is not necessary to have mediation by mental representations of the physical world (such as conceptions about pitch or speed) in order to experience something as urgent or to act on it appropriately (Gibson, 1979). A related possibility is that the experience of urgency is based upon familiarity with probabilistic cues inherent in emotional speech. Listeners appear to perceive elevations in speech rate, pitch, or both not in terms of acoustics but in terms of a speaker's level of arousal (Scherer, 1986). In sum, it is possible that perceptual mediation is not necessary to experience urgency and that a single monotonic function can be used to characterize both rate-based predictors. 
In this study, we addressed the role of perceptual mediation and whether psychometric functions concerning different rate-based predictors of urgency are truly independent. This question was assessed by developing a continuum of pulse-train stimuli that varied only in rate (Miller and Taylor, 1948). At the low end of the continuum, pulses were easily resolved, with changes in rate corresponding to speed (or tempo). At the high end of the continuum, pulses fused together perceptually leading to a tonal sensation with a clear pitch level. To our knowledge, this is the first study of urgency incorporating phenomenological changes in speed and pitch along a single rate-based continuum.

\section{Urgency scaling experiment}

A magnitude estimation task was conducted to assess the experienced urgency of pulse trains that varied in rate between 3.13 and $200 \mathrm{~Hz}$.

\subsection{Participants}

Thirty participants ( 20 women and $10 \mathrm{men}$ ) with normal hearing were asked to provide magnitude estimations. The mean age of participants was 21 years ( $\mathrm{SD}=1.9$ years). The procedures were approved by the Wilfrid Laurier University Research Ethics Board and all participants gave informed consent.

\subsection{Stimuli and apparatus}

Pulses (bursts of white noise) were interleaved with silent intervals (of equal duration) at varying rates of repetition. The rate of the resulting pulse train was thus determined by the interonset-interval of the noise burst. Rates were equally spaced on a log scale ranging from 3.13 to $200 \mathrm{~Hz}$. Sounds were presented in a double-walled sound-attenuated chamber over headphones (Sony Stereo Headphones MDR-XD200) at a presentation level of $70 \mathrm{~dB}$ SPL.

\subsection{Procedure}

Each sound was presented for a duration of $2.5 \mathrm{~s}$ with the order of sounds being independently randomized for each participant. Participants judged the experienced urgency of each sound using a free-modulus magnitude estimation task (Stevens, 1975) in which participants are asked to assign a numerical value to each sound. The absence of a fixed scale (e.g., 1 to 7 ) improves sensitivity of measurement by encouraging participants to actively compare each response to all other responses. Participants' responses initiated the following trial and each session lasted approximately $15 \mathrm{~min}$.

\subsection{Results and discussion}

Responses of each participant were first standardized (converted to z-scores) in order to avoid biases arising from outliers (e.g., a participant that used an unusually wide range of numerical ratings). Although the absolute values of the reported standardized scores are not meaningful, the relative values should reflect real differences experienced by the sample of participants.

Standardized ratings of urgency across the sound frequencies tested may be seen in Fig. 1. Urgency ratings were not equivalent across pulse rates, $\mathrm{F}(12,348)=5.26, p<0.0001$. Trend analysis revealed that the linear trend was not significant, $F(1,29)=1.16$, n.s. and the quadratic trend was only marginally significant, $\mathrm{F}(1,29)=4.39, p<0.05$. The best-fitting polynomial trend was cubic, $\mathrm{F}(1,29)=47.18, p<0.0001$, indicating that there are two changes in the directionality of the relationship between urgency and pulse rate (for the range of pulse rates tested). The strong cubic trend is a highly unusual scaling result and suggests the possibility of two underlying power functions. The first, peaking at $17.68 \mathrm{~Hz}$, likely corresponds to the upper limit for which sequential pulses may be resolved (i.e., infrapitch). For higher pulse rates (e.g., beyond $35.36 \mathrm{~Hz}$ ), sequential pulses appear to fuse and give rise to a clear pitch percept (see also Fitzgerald and Wright, 2005; Miller and Taylor, 1948; Pollack, 1969). Intermediate pulse rates would have had a repetition rate that was too high to resolve independent pulses but too 


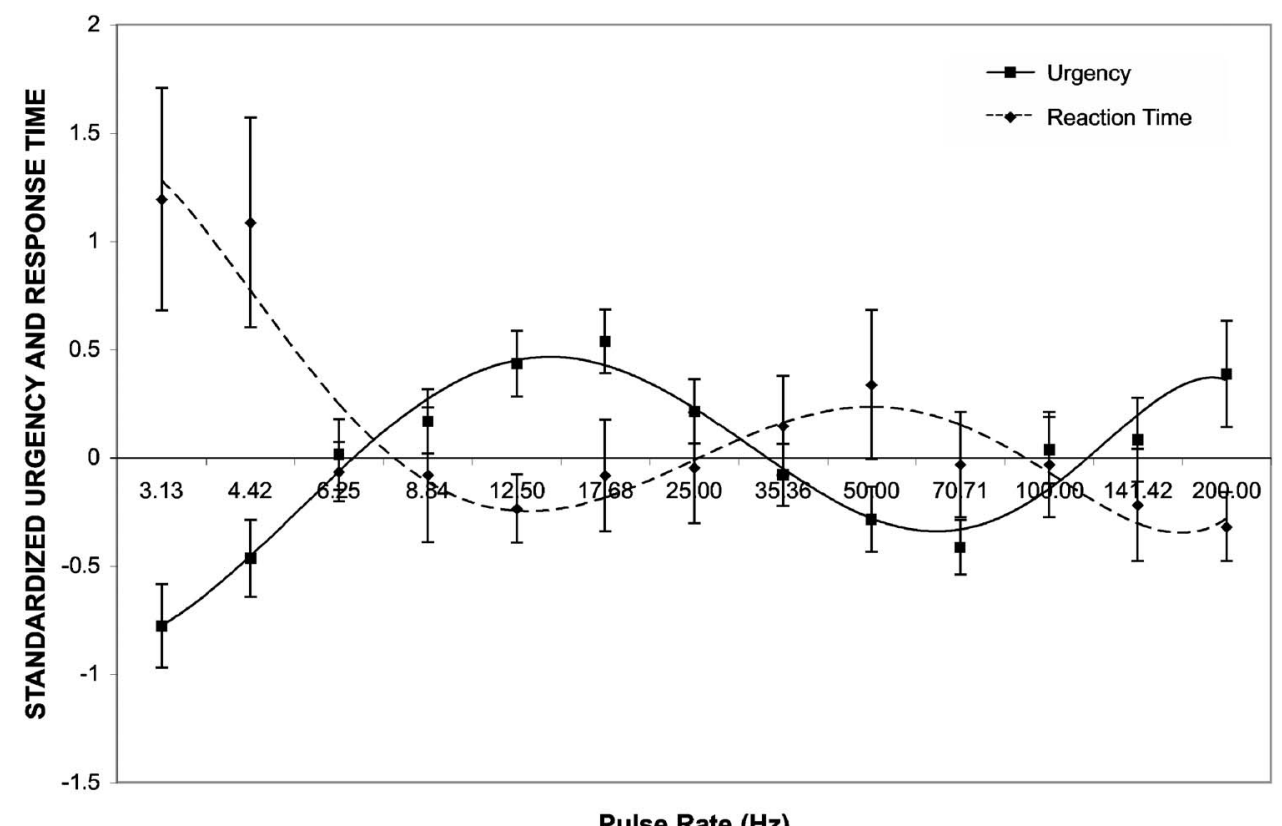

Fig. 1. Mean standardized urgency ratings and mean standardized response times for each pulse rate. Error bars represent standard error.

low to yield a clear pitch percept. These results strongly suggest that the experience of urgency in response to an auditory warning is mediated by perceptual constructs.

Following previous urgency research (Hellier et al. 1993; 1999), urgency ratings were mapped using an application of Stevens Power Law (Stevens, 1975), Eq. (1):

$$
U=k f^{m}
$$

where $U$ is the judged urgency (in log units), $f$ is the frequency or pulse rate (in log units), and $k$ and $m$ are the intercept and slope of the line (exponent) of best fit.

For the range of pulse rates from 3.13 to $17.68 \mathrm{~Hz}$ ("infrapitch region"), the power function exponent was 1.10. For the range of pulse rates between 35.36 and $200 \mathrm{~Hz}$ ("pitch region"), the exponent was 0.32 . The relative and absolute value of these exponents are comparable to those reported by Hellier et al. (1999) for the warning parameters of speed and pitch.

\section{Response time experiment}

In order to verify whether the effects of the urgency mapping experiment reflected the experience of urgency, Experiment 2 asked participants to perform a tracking task (Makeig and Jolley, 1996) while occasionally responding to an auditory warning as quickly as possible. If the urgency ratings were based on experienced urgency, there should be an inverse pattern of response times for the same stimuli.

\subsection{Participants}

Nine participants (six women and three males) with normal hearing were asked to participate in the tracking experiment. The mean age of participants was $22.8(\mathrm{SD}=1.7 \mathrm{y})$. The procedures were approved by the Wilfrid Laurier University Research Ethics Board and all participants gave informed consent. 


\subsection{Stimuli and apparatus}

The tracking task was presented on a PC computer with a standard video display and roller mouse using COMPTRACK software (Makeig and Jolley, 1995). The pulse-train stimuli and apparatus were identical to those described in Sec. 2.2.

\subsection{Procedure}

The tracking task required participants to use a computer mouse to keep a circular cursor within a target circle by compensating for simulated buffeting and gravitational forces that acted upon it. Participants were asked to simultaneously listen for the auditory warning and to respond to it as quickly as possible whenever it was heard by pressing a button on a button box (i.e., not the mouse button).

\subsection{Results and discussion}

Standardized response times may be observed in Fig. 1. All participants displayed a high degree of vigilance in the tracking task. An ANOVA confirmed that response times varied with regard to pulse rate, $\mathrm{F}(12,96)=2.27, p<0.05$. Average response times to pulse trains were shortest for pulse rates of $17 \mathrm{~Hz}(385.36 \mathrm{msec})$ and $200 \mathrm{~Hz}(384.14 \mathrm{msec})$. By comparison, response times were much longer for the lowest pulse rates in the infrapitch region $(3.13 \mathrm{~Hz}, 524.17 \mathrm{msec}$; $4.42 \mathrm{~Hz}, 546.04 \mathrm{msec})$ as well as for the lowest pulse rates in the pitch region $(35.36 \mathrm{~Hz}$, $435.46 \mathrm{msec} ; 50 \mathrm{~Hz}, 483.04 \mathrm{msec}$ ). It is important to note that these response time differences were obtained in a fully alert low-cognitive load condition. The response time differences would likely be further exaggerated under less optimal cognitive conditions. Nonetheless, the correlation between mean urgency ratings and mean response times was negative and highly significant, $r(11)=-0.84, p<0.0001$, suggesting that the polynomial function obtained for urgency reflected the experience of urgency, rather than a conscious integration of discrete perceptual mappings.

\section{Pitch strength scaling experiment}

To verify the phenomenological descriptions of pitch regions described in Sec. 2.4, pulse trains were subjected to a magnitude estimation task involving pitch strength; that is, the extent to which sounds give rise to a pitch percept. We expected perceived pitch strength to be at baseline (equal to pitch strength of white noise) between 17.68 and $35.36 \mathrm{~Hz}$ (transition region) and to rise sharply beyond $35.36 \mathrm{~Hz}$ (pitch region).

\subsection{Participants}

Twenty-seven participants ( 21 women and 6 men) with normal hearing were asked to provide magnitude estimations. The mean age of participants was 19.13 years (SD 1.5 years).

\subsection{Stimuli and apparatus}

The stimuli and apparatus were identical to that described in Sec. 2.2 with the exception that participants heard stimuli through Sennheiser HD 580 Precision headphones and the addition of a standard sound consisting of $2.5 \mathrm{~s}$ broadband white noise presented at $70 \mathrm{~dB}$ SPL.

\subsection{Procedure}

Participants judged pitch strength of each pulse train relative to the standard using a freemodulus magnitude estimation task. The following specific instructions were provided in order to clarify the objective of the task: "We have arbitrarily assigned the standard a pitch strength value of 10 . You will be judging the pitch strength of the comparison sound in each presentation relative to the standard. If the comparison sound appears to be of the same pitch strength as the standard, then you would give a response of 10. If the comparison appears to have three times the pitch strength of the standard, then you would give a response of 30 , and so on." In total, each participant judged all sounds four times each. Sessions lasted approximately 25 min. 


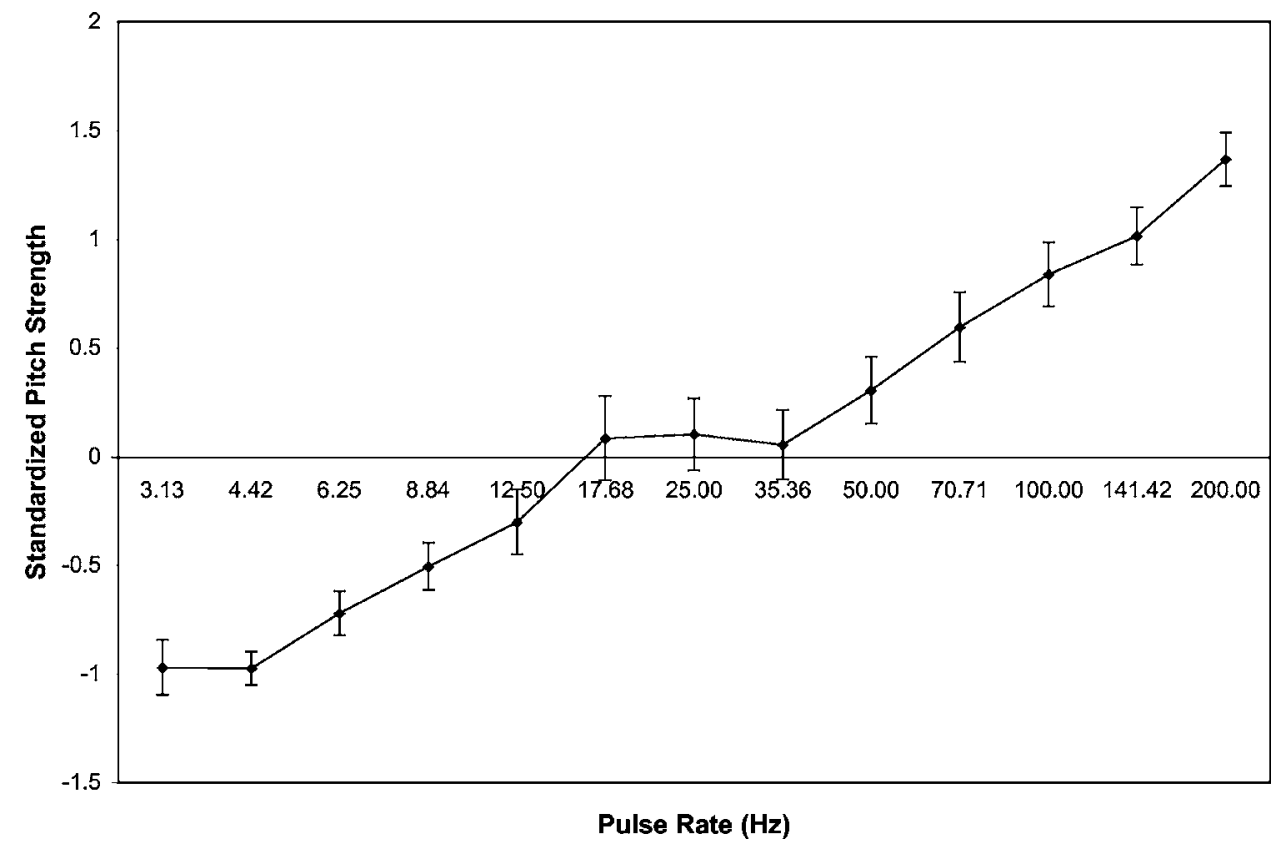

Fig. 2. Mean standardized pitch strength ratings for each pulse rate. Error bars represent standard error.

\subsection{Results and discussion}

The mean standardized ratings of pitch strength varied across pulse rates, $\mathrm{F}(12,312)=33.68$, $p<0.0001$. Figure 2 shows that there was no change in pitch strength between 17.68 and $35.36 \mathrm{~Hz}$ (transition region). Pulse trains in this region had pitch strength that corresponded to that of an extended sample of white noise-i.e., pitch was ambiguous. There was a sharp rise beyond $35.36 \mathrm{~Hz}$, which is suggestive of a fusing of pulses leading to a tonal sensation with an increasingly clear pitch level with increasing pulse rate. Examination of Fig. 2 also reveals a drop off in pitch strength below $17.68 \mathrm{~Hz}$ (infrapitch region). The drop-off is somewhat unexpected given that pulse trains in this region do not appear to fuse and may reflect metacognitive awareness of the transition from infrapitch to pitch with increasing pulse rate.

These scaling results are consistent with more objective tests of low-frequency pitch perception (Pressnitzer et al., 2001; Russo et al., 2007) and with pitch matching experiments involving pulse trains (Miller and Taylor, 1948). The latter study showed that the frequency of interruption of a white-noise pattern only corresponded to the matched pitch of a pure tone for frequencies above $40 \mathrm{~Hz} .^{1}$

\section{Conclusions}

The current study has identified two local maxima in a nonmonotonic (polynomial) function relating pulse rate to perceived urgency. The first maximum at $17.68 \mathrm{~Hz}$ corresponds to the positive correlation known to exist between speed and urgency (Guillaume et al., 2003; Hellier et al., 1993; 1995; 1999). Sounds in this region had very low pitch strength (i.e., infrapitch) consisting of a sequence of resolved pulses. The dip beyond $25 \mathrm{~Hz}$ likely reflects the transition from independent to fused pulses; that is, a transition from a sensation involving high-speed and no pitch to a sensation involving no speed and clear pitch. The second maximum, at $200 \mathrm{~Hz}$ reflects the positive correlation known to exist between pitch and urgency (Guillaume et al., 2003; Hellier et al., 1993; 1995; 1999). These findings suggest that the influence of rate-based cues on the experience of urgency in auditory warnings is mediated by perceptual constructs. 


\section{Acknowledgments}

We wish to thank Farina Pinnock for research assistance. Research was supported by Discovery grants awarded to each author from the Natural Science and Research Council of Canada and a Research Fellowship from Wilfrid Laurier University awarded to the second author.

\footnotetext{
${ }^{1}$ We asked 23 participants with little to no music training do a pure-tone matching experiment with our pulse-train stimuli. Tuning accuracy improved as a function of pulse rate. Errors were consistently large for pulse rates below $35.34 \mathrm{~Hz}$ but fell to within about two semitones for pulse rates above $70 \mathrm{~Hz}$.
}

Edworthy, J., Loxley, S., and Dennis, I. (1991). "Improving auditory warning design: Relationship between warning sound parameters and perceived urgency," Hum. Factors 33, 205-231.

Fitzgerald, M. B., and Wright, B. A. (2005). "A perceptual learning investigation of the pitch elicited by amplitudemodulated noise," J. Acoust. Soc. Am. 118, 3794-3803.

Gibson, J. J. (1979). “The ecological approach to visual perception (Houghton Mifflin: Boston, MA).

Guillaume, A., Pellieux, K., Chastres, V., and Drake, C. (2003). "Judging the urgency of nonvocal auditory warning signals: Perceptual and cognitive processes,” J. Exp. Psychol., Appl. 9, 196-212.

Hellier, E. J., and Edworthy, J. (1999). "On using psychophysical techniques to achieve urgency mapping in auditory warnings," Appl. Ergon 30, 167-171.

Hellier, E. J., Edworthy, J., and Dennis, I. (1995). "A comparison of different techniques for scaling perceived urgency," Ergonomics 38, 659-670.

Hellier, E. J., Edworthy, J., and Dennis, I. (1993). "Improving auditory warning design: Quantifying and predicting the effects of different warning parameters on perceived urgency," Hum. Factors 35, 693-706.

Makeig, S., and Jolley, K. (1995). "COMPTRACK: A compensatory tracking task for monitoring alertness," In Technical Document No. 93-3C, Naval Health Research Center, San Diego, CA.

Miller, G. A., and Taylor, W. (1948). "The perception of repeated bursts of noise," J. Acoust. Soc. Am. 20, 171-182. Neuhoff, J. G. (2004). "Ecological psychoacoustics: Introduction and history," edited by J. G. Neuhoff, Ecological Psychoacoustics ( Academic Press, New York), pp. 4-12.

Patterson, R. D. (1990). "Auditory warning sounds in the work environment," Philos. Trans. R. Soc. London, Ser. B 327, 485-492.

Pollack, I. (1969). "Periodicity pitch for interrupted white noise-Fact or artifact?," J. Acoust. Soc. Am. 45, 237238.

Pressnitzer, D., Patterson, R. D., and Krumbholz, K. (2001). “The lower limit of melodic pitch,” J. Acoust. Soc. Am. 109, 2074-2084.

Russo, F. A., Cuddy, L. L., Galembo, A., and Thompson, W. F. (2007). "Sensitivity to tonality across the pitch range," Perception 36, 781-790.

Scherer, K. R. (1986). "Vocal affect expression: A review and a model for future research," Psychol. Bull. 99, $143-165$.

Stevens, S. S. (1975). "Psychophysics: Introduction to its perceptual, neural, and social prospects," (Wiley, New York).

Stockhausen, Karlheinz. (1990/1971). "Stockhausen on Music: Public lectures in English from 1971 transcribed by Robin Maconie,” (Marion Boyars, London, New York). 